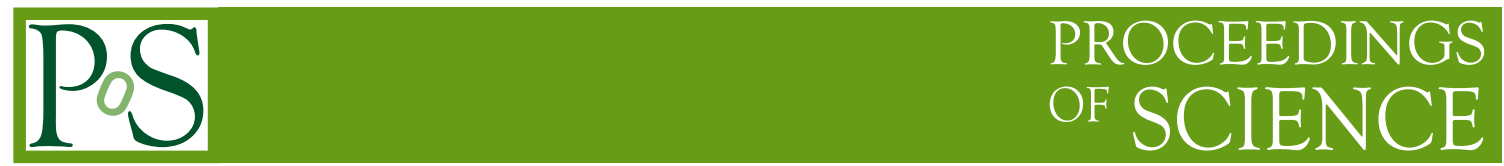

\title{
Electroweak and Top Physics in the Forward Region
}

\section{Sestini on behalf of the LHCb collaboration ${ }^{a}$ \\ a INFN-Padova}

E-mail: lorenzo.sestini@cern.ch

The LHCb detector at the LHC offers unique coverage of forward rapidities, allowing the experiment to play an important role in measurements of Standard Model processes. Measurements of $\mathrm{W}, \mathrm{Z}$, top and jet production at $\mathrm{LHCb}$ will be presented, and future prospects will be discussed. These measurements can be used to test the perturbative QCD and electroweak theories in a phase space complementary with respect to ATLAS nad CMS, and to probe the parton distribution functions in a region unexplored by other experiments.

40th International Conference on High Energy physics - ICHEP2020

July 28 - August 6, 2020

Prague, Czech Republic (virtual meeting) 


\section{Introduction}

$\mathrm{LHCb}$ is a forward spectrometer, initially designed for $b$ - and $c$-quarks physics. Within the LHC experiments, LHCb alone provides precision coverage in the forward region of $p p$ collisions corresponding to the $2 \leq \eta \leq 5$ pseudorapidity range. In both LHC Run I and Run II, LHCb demonstrated its capability as a general purpose forward detector. Precision measurements involving $W$ and $Z$ bosons, top quarks, $b$ - and $c$-jets are important tests of perturbative QCD and electroweak theory, within the standard model (SM). Moreover, they can be used to probe the parton distribution functions (PDFs): at LHCb two different regions are available in the $x-Q^{2}$ phase space, where $x$ is the momentum fraction of proton momentum taken by the parton and $Q^{2}$ is the transferred momentum between the partons that participate to the hard interaction. The region at low $x$ and high $Q^{2}$ is still unexplored, and inaccessible by other experiments at the LHC.

\section{Measurements of the electroweak bosons production}

LHCb performed the measurement of the $Z$ boson production cross-section in several final states: $Z \rightarrow \mu^{+} \mu^{-}, Z \rightarrow e^{+} e^{-}, Z \rightarrow \tau^{+} \tau^{-}$and $Z \rightarrow b \bar{b}$.

In the $Z \rightarrow \mu^{+} \mu^{-}\left(Z \rightarrow e^{+} e^{-}\right)$measurement at $\sqrt{s}=13 \mathrm{TeV}$ [1], muons (electrons) of the final state are selected with $2<\eta<4.5(4.25)$ and transverse momentum $p_{\mathrm{T}}>20 \mathrm{GeV} / c$. In addition, the dilepton invariant mass must be in the $60<m_{l l}<120 \mathrm{GeV} / c^{2}$ range. The low background contamination is evaluated using simulation and data-driven techniques (i.e. by considering opposite sign-leptons). We obtained purities of $99.2 \%$ and $92.2 \%$ for the muon and electron final states, respectively. The $Z \rightarrow \tau^{+} \tau^{-}$decays have been reconstructed in events at $\sqrt{s}=8 \mathrm{TeV}$ by selecting different streams of $\tau$ decays: electron, muon, one-hadron or three-hadrons. The background has been estimated through simulation and data-driven techniques. The measured $Z \rightarrow \mu^{+} \mu^{-}$, $Z \rightarrow e^{+} e^{-}$and $Z \rightarrow \tau^{+} \tau^{-}$cross sections are shown in Fig. 1, compared with theoretical predictions obtained at next-to-next-to-leading-order (NNLO).
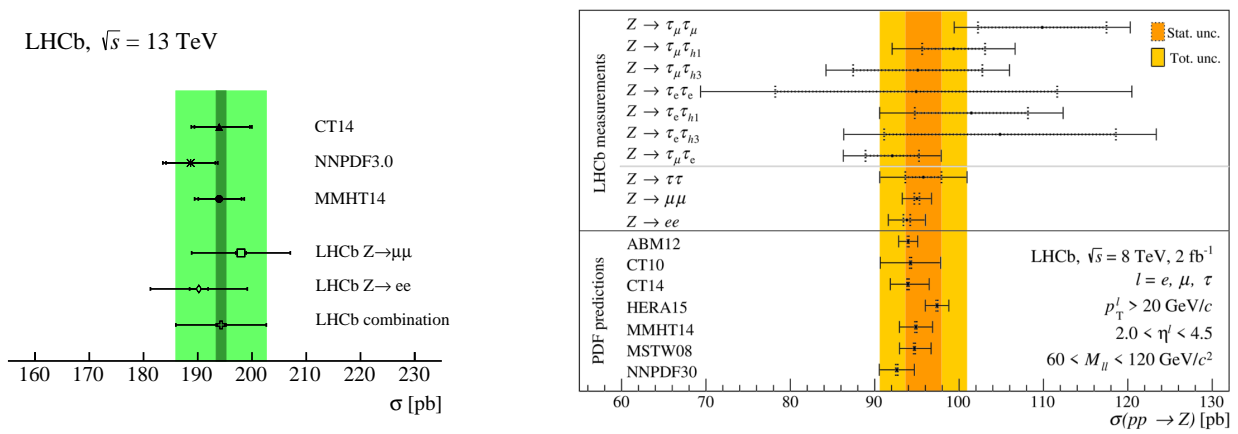

Figure 1: (Left) $Z \rightarrow \mu^{+} \mu^{-} / e^{+} e^{-}$cross-sections in collisions at $13 \mathrm{TeV}$, compared with NNLO predictions. (Right) $Z \rightarrow \tau^{+} \tau^{-}$cross-sections in several streams of $\tau$ decays (black bars) and combined result (orange/yellow bars) in collisions at $8 \mathrm{TeV}$, compared with predictions.

The decay $Z \rightarrow b \bar{b}$ has been reconstructed in $p p$ collision data at $\sqrt{s}=8 \mathrm{TeV}$ [8]. The fiducial region of the measurement is defined by $b$-jets with $2.2<\eta<4.2, p_{\mathrm{T}}>20 \mathrm{GeV} / c$ and with a dijet 
invariant mass $\left(m_{j j}\right)$ in the range $45<m_{j j}<165 \mathrm{GeV} / c^{2}$. At LHCb jets are recostructed with the anti- $k_{T}$ algorithm using a radius parameter of 0.5 , and $b$-jets are identified with the algorithm described in [7] where a secondary vertex (SV) consistent with the decay of a beauty or charm hadron is required inside the jet cone. In this measurement a signal and a control region are defined using the kinematic of additional jets in the events, and the $m_{j j}$ distributions in these two regions are simultaneously fitted to determine the $Z \rightarrow b \bar{b}$ and multi-jets background yields. The background subtracted dijet invariant mass distributions in signal and control regions are shown in Fig. 2. A $Z \rightarrow b \bar{b}$ production cross-section of $332 \pm 46$ (stat.) \pm 59 (syst.) $\mathrm{pb}$ is obtained and it is in agreement with the next-to-leading (NLO) prediction.
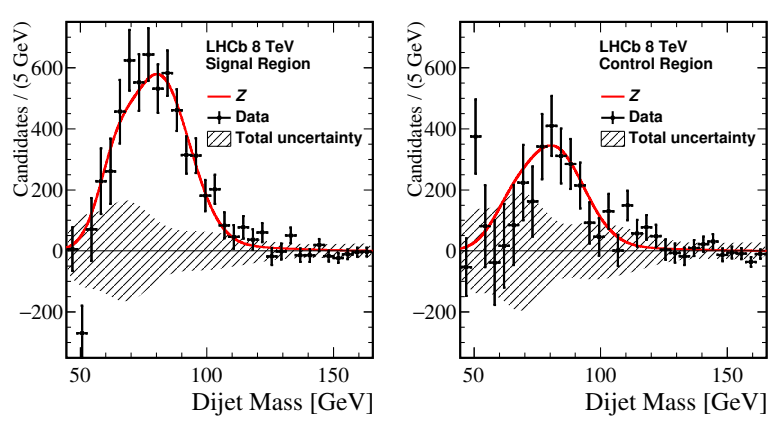

Figure 2: Background-subtracted $m_{j j}$ distributions in the signal region (left) and in the control region (right). The uncertainty band in the background only hypothesis is also shown.

LHCb performed the measurement of the $W \rightarrow \mu v$ and $W \rightarrow e v$ boson production cross-section in $p p$ collisions at $\sqrt{s}=8 \mathrm{TeV}[3,4]$. Muons (electrons) candidates must have $2<\eta<4.5(4.25)$, $p_{\mathrm{T}}>20 \mathrm{GeV} / c$ and a certain degree of isolation with respect to other particles. The purity of the $W^{ \pm}$samples has been obtained with fits to the lepton $p_{\mathrm{T}}$ distribution. The measured cross-sections are in agreement with NNLO predictions calculated with different PDFs sets, and the ratio between $W \rightarrow \mu v$ and $W \rightarrow e v$ cross-sections is compatible with 1 .

\section{Measurements of the electroweak bosons production in association with jets}

The cross-section measurement of the $W$ and $Z$ boson production in association with a jet has been performed using $p p$ collisions at $\sqrt{s}=8 \mathrm{TeV}$ [5]. The $W$ and $Z$ bosons are selected in the $W \rightarrow \mu^{+} \nu^{-}$and $Z \rightarrow \mu^{+} \mu^{-}$decays as explained above, and the associated jet is required to have $p_{\mathrm{T}}>20 \mathrm{GeV} / c$ and $2.2<\eta<4.2$.

The purity of the $W^{+}+$jet and $W^{-}+$jet samples has been evaluated by fitting the distribution of the muon isolation, defined as the $p_{\mathrm{T}}$ of the muon divided by the $p_{\mathrm{T}}$ of the jet that contains the signal muon. The purity of the $Z+$ jet sample has been evaluated by using background-enhanced data samples and simulation samples.

The cross-sections have been measured as a function of the jet and muon kinematics: as examples the $Z+$ jet and $W^{+}\left(W^{-}\right)+$jet cross-sections as a function of the jet $\eta$ are shown in Fig. 3, and they are compatible with NLO predictions. 

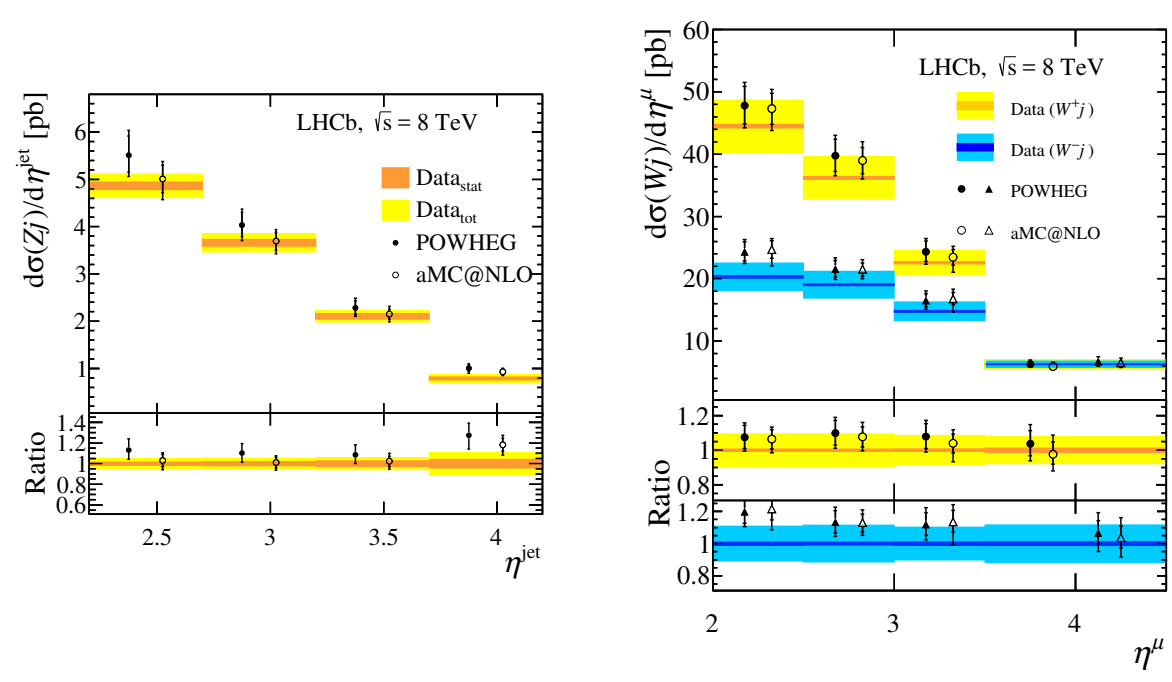

Figure 3: $Z+$ jet cross-section as a function of the jet $p_{\mathrm{T}}$ (left) and the $W+$ jet cross-section as a function of the muon $\eta$ (right). NLO predictions are also shown.

LHCb performed the observation of the $W+b \bar{b}$ production and the first measurement of the $W+c \bar{c}$ process, in data collected at $\sqrt{s}=8 \mathrm{TeV}[6]$. Using the same sample, the $t \bar{t}$ cross-section in the forward region has been also measured. The $W$ boson is selected in the muon or electron final state, jets are selected with $p_{\mathrm{T}}>12.5 \mathrm{GeV} / c$ and $2.2<\eta<4.2$. SVs are also required inside the jets. The number of $W^{+}+b \bar{b}, W^{-}+b \bar{b}, W^{+}+c \bar{c}, W^{-}+c \bar{c}$ and $t \bar{t}$ events is extracted by simultaneously fitting the distribution of $m_{j j}$, the distributions of two multivariate discriminators, one for each selected jet, which exploit properties of the SVs to separate $b$ from $c$, and the distribution of one multivariate discriminator that separates the $W+b \bar{b}$ process from the $t \bar{t}$ process. The measured cross-sections, shown in Fig. 4, are in agreement with NLO predictions.

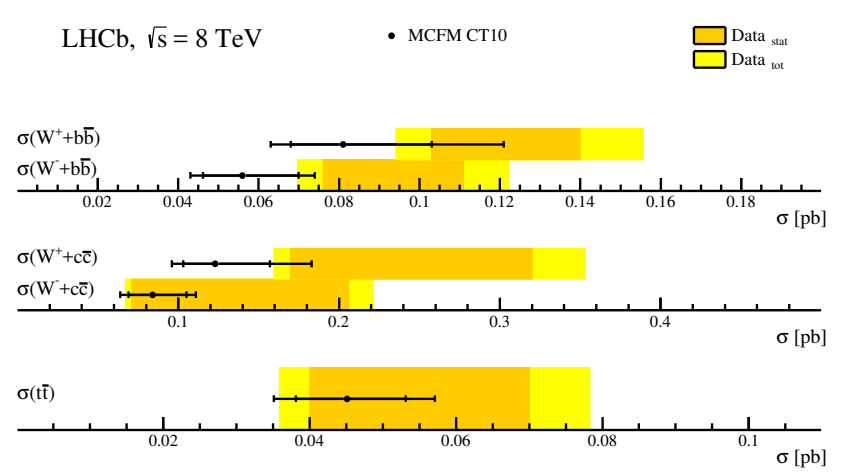

Figure 4: $W^{+}+b \bar{b}, W^{-}+b \bar{b}, W^{+}+c \bar{c}, W^{-}+c \bar{c}$ and $t \bar{t}$ measured cross-sections (orange/yellow bars) compared with predictions (black bars). 


\section{Measurements of the $t \bar{t}, b \bar{b}$ and $c \bar{c}$ production in the forward region}

The measurement of the $t \bar{t}$ production in the $\mu e b$ final state has been performed using $1.93 \mathrm{fb}^{-1}$ of integrated luminosity at $\sqrt{s}=13 \mathrm{TeV}$ [9]. The two leptons are required to have $p_{\mathrm{T}}>20 \mathrm{GeV} / c$ and a separation of $\Delta R=\sqrt{(\Delta \eta)^{2}+(\Delta \phi)^{2}}>0.1$, where $\Delta \phi$ is the azimuthal angle difference. The $b$-jet is required to have $p_{\mathrm{T}}>20 \mathrm{GeV} / c$ and a separation of $\Delta R>0.5$ with respect to the leptons. In the analysis the background has been evaluated with simulation and data-driven techniques: a signal purity of about $85 \%$ is expected. A total of 44 events have been selected, and the signal yield has been obtained by subtracting the background. The measured invariant mass distribution of the $\mu e b$ system is shown in Fig. 5. By correcting for the selection efficiencies in the fiducial region, a cross section of $126 \pm 19$ (stat) \pm 16 (syst) \pm 5 (lumi) fb has been measured and it is compatible with the NNLO prediction. The systematic uncertainty is dominated by the knowledge of the $b$-tagging efficiency.
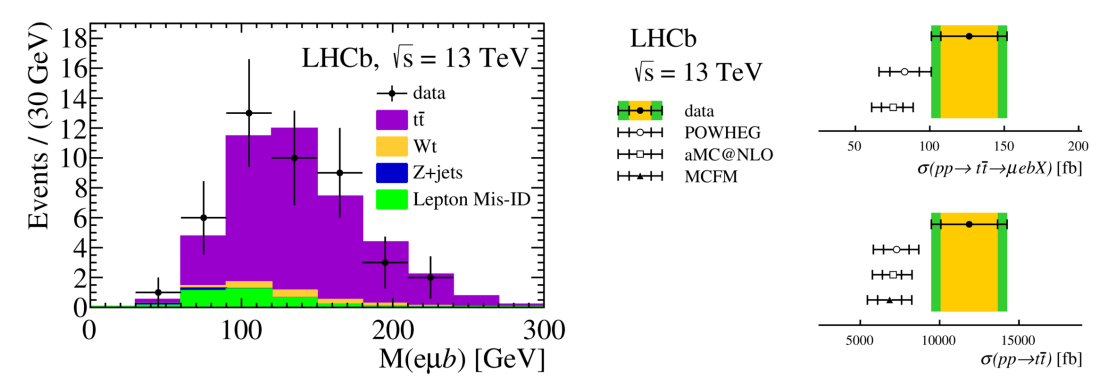

Figure 5: Invariant mass distribution of $\mu e b$ candidates (left), measured $t \bar{t}$ cross-section in the forward region (top right, yellow bar is the statistical uncertainty and green is the systematic one) and extrapolated in the full acceptance (down right).

The production of forward $b$-jet and $c$-jet pairs in the forward region has been measured with 2 $\mathrm{fb}^{-1}$ of integrated luminosity at $\sqrt{s}=13 \mathrm{TeV}$ [10]. In this analysis the differential $b \bar{b}$ - and $c \bar{c}$-dijet cross-sections as a function of leading jet $p_{\mathrm{T}}$ and $\eta$, rapidity difference and $m_{j j}$ are determined. The fiducial region of the measurement is defined by two jets with $p_{\mathrm{T}}>20 \mathrm{GeV} / c, 2.2<\eta<4.2$ and $\Delta \phi>1.5$. SVs are required in both jets to select heavy-flavour. The sample selected in this way is formed by $b \bar{b}, c \bar{c}$ and background processes where one or two light jets are misidentified. The flavour composition is measured by fitting the distribution of multivariate classifiers that have in input variables related to the two SVs. An unfolding technique is applied to correct for detector effects and measure the differential cross-section, which are shown, as a function of $m_{j j}$, in Fig. 6 together with predictions.

\section{Conclusions}

An overview of the measurements performed by $\mathrm{LHCb}$ including electroweak bosons, with or without associated jets, and heavy flavour productions have been presented: they are competitive and complementary with the corresponding measurements of the other LHC experiments. These measurements are important tests of the SM and they can provide constraints to PDFs in a unique 

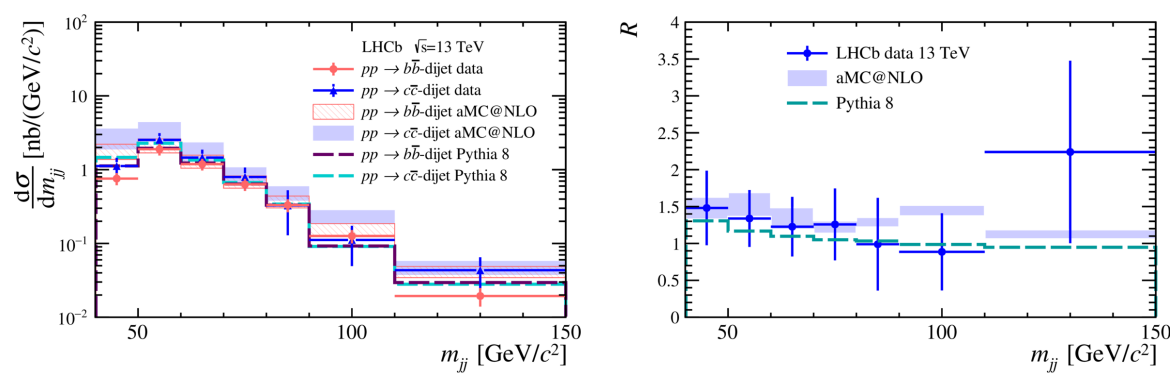

Figure 6: Differential $b \bar{b}$ - and $c \bar{c}$-dijet cross sections (left) and $c \bar{c}$ to $b \bar{b}$ ratio as a function of $m_{j j}$.

kinematic region. In the future, by collecting more data, it will be possible to increase the statistics of the $t \bar{t}$ sample. Moreover the reduction of the systematics on the tagging efficiency using dedicated calibration samples will allow to improve the precision of $b$ - and $c$-jets measurements. The precision measurement of fundamental SM parameters like the $W$ boson mass and the electroweak mixing will be also possible.

\section{References}

[1] LHCb collaboration, Measurement of the forward $Z$ boson production cross-section in $p p$ collisions at $\sqrt{s}=13 \mathrm{TeV}$, JHEP 09 (2016) 136.

[2] LHCb collaboration, Measurement of $Z \rightarrow \tau^{+} \tau^{-}$production in proton-proton collisions at $\sqrt{s}$ $=8 \mathrm{TeV}$, JHEP 09 (2018) 159 .

[3] LHCb collaboration, Measurement of forward $W$ and $Z$ boson production in pp collisions at at $\sqrt{s}=8 \mathrm{TeV}$, JHEP 01 (2016) 155 .

[4] LHCb collaboration, Measurement of forward $W \rightarrow$ ev production in pp collisions at $\sqrt{s}=8$ TeV, JHEP 10 (2016) 030.

[5] LHCb collaboration, Measurement of forward $W$ and $Z$ boson production in association with jets in proton-proton collisions at $\sqrt{s}=8 \mathrm{TeV}$, JHEP 05 (2016) 131.

[6] LHCb collaboration, Measurement of forward $t \bar{t}, W+b \bar{b}$ and $W+c \bar{c}$ production in pp collisions at $\sqrt{s}=8 \mathrm{TeV}$, Phys. Lett. B767 (2017) 110.

[7] LHCb collaboration, Identification of beauty and charm quark jets at LHCb, JINST 10 (2015) P06013.

[8] LHCb collaboration, Measurement of forward $Z \rightarrow b \bar{b}$ production in pp collisions at $\sqrt{s}=8$ TeV, Phys. Lett. B776 (2017) 430-439.

[9] LHCb collaboration, Measurement of forward top pair production in the dilepton channel in pp collisions at $\sqrt{s}=13 \mathrm{TeV}$, JHEP 08 (2018) 174 .

[10] LHCb collaboration, Measurement of differential $b \bar{b}$ - and $c \bar{c}$-dijet cross-sections in the forward region of pp collisions at $\sqrt{s}=13 \mathrm{TeV}$, arxiv:2010.09437. 